\title{
Two-Dimensional Electrophoretic Separation of Proteins Using Poly(methyl methacrylate) Microchips
}

\author{
Hamed Shadpour ${ }^{1}$ and Steven A. Soper ${ }^{\star 1,2}$ \\ ${ }_{1}^{1}$ Department of Chemistry and Center for BioModular Multi-Scale Systems, Louisiana State University, \\ Baton Rouge, Louisiana 70803, '2Department of Mechanical Engineering, Louisiana State University, \\ Baton Rouge, Louisiana 70803
}

\section{SUPPORTING INFORMATION}




\section{EXPERIMENTAL SECTION}

Fabrication of PMMA microchips. The PMMA microchips were replicated from a brass master that was fabricated using high precision micromilling and hot embossing following the procedure that is described herein. A 0.25 " thick brass sheet (alloy 353 engravers brass, McMaster-Carr, Atlanta, GA) was machined into a $12 \mathrm{~cm}$ diameter circular plate. Plate flatness was typically within $\pm 2 \mu \mathrm{m}$ and was verified by a surface profiler (Tencor P11, KLA-Tencor, San Jose, CA). Microstructures were milled onto the brass plate with a micro-milling machine (KERN MMP 2522, KERN Micro-und Feinwerktechnik GmbH \& Co. KG, Germany). 500, 200, 100, 50, and $25 \mu \mathrm{m}$ solid carbide milling bits were used (McMaster-Carr or Quality Tools, Hammond, LA) for micro-milling, which was carried out at 40,000 rpm feed rates that were optimized for maximum machining speed and quality of the microstructures. Feed rates were dependent on the size of the milling tool and were typically in the range of $200 \mathrm{~mm} / \mathrm{min}$ for the $500 \mu \mathrm{m}$ milling bit, $100-150 \mathrm{~mm} / \mathrm{min}$ for the $200 \mu \mathrm{m}$ bit, $50-75 \mathrm{~mm} / \mathrm{min}$ for the $100 \mu \mathrm{m}$ bit, $10-20$ $\mathrm{mm} / \mathrm{min}$ for the $50 \mu \mathrm{m}$ bit, and $2-4 \mathrm{~mm} / \mathrm{min}$ for the $25 \mu \mathrm{m}$ bit. A typical milling cycle consisted of a precut of the entire surface with the $500 \mu \mathrm{m}$ milling bit to ensure parallelism between both faces of the brass plate, a rough milling of the microstructures using the 500 or $200 \mu \mathrm{m}$ milling bit, and a finishing cut with the required smaller diameter milling bit. In the final step, burrs produced at the top of the microstructures were removed by mechanical polishing. Polishing was performed on a $3 \mu \mathrm{m}$ grain size polishing paper (Fibrmet Discs - PSA, Buehler, Lake Bluff, IL) followed by polishing on a polypropylene cloth (Engis, Wheeling, IL) with a $1 \mu \mathrm{m}$ diamond suspension (Metadi Diamond Suspension, Buehler).

Before hot embossing, the PMMA wafers were kept in an oven $\left(80^{\circ} \mathrm{C}\right)$ overnight to remove any absorbed water. During embossing, the mold master was heated $\left(160^{\circ} \mathrm{C}\right)$ and pressed into the polymer wafer with a pressure of $1,100 \mathrm{psi}$ for $410 \mathrm{~s}$. The embossing plates were then retracted and the polymer substrate removed and cooled to room temperature. The entire fluidic network was sealed with a thin 
polymer cover plate by clamping the plastic pieces between two glass plates and thermally annealing in a $\mathrm{GC}$ oven $\left(120^{\circ} \mathrm{C}, 20 \mathrm{~min}\right) .^{1}$

\section{RESULTS AND DISCUSION}

Zonal Spreading due to the coupled 2D process. Loss of peak capacity due to zonal dispersion induced by the on-line coupled two-dimensional (2D) separation can result from either the interface of the twodimensions of the 2D separation, incompatibility of the individual separation dimensions or "parking" of zones for a serial multi-dimensional separation as that used herein. We evaluated the effects of zonal spreading due to longitudinal diffusion in the first dimension during the development phases of the separations in the second dimension. This was accomplished by calculating the height equivalent of a theoretical plate for longitudinal diffusion only $\left(\mathrm{H}_{\mathrm{D}}\right)$ of the 1D SDS $\mu$-CGE dimension and comparing that value to $\mathrm{H}_{\text {тот }}$ secured from the complete $2 \mathrm{D}$ separation. A representative diffusion coefficient for proteins in a sieving matrix as used herein was taken as $\sim 10^{-8} \mathrm{~cm}^{2} \mathrm{~s}^{-1}$, which is the measured diffusion coefficient of cytochrome $\mathrm{C}$ in polyacrylamides ${ }^{2}$ resulting in $\mathrm{H}_{D}=2.6 \times 10^{-6} \mathrm{~cm}\left(\mathrm{H}_{D}=2 \mathrm{Dt} / \mathrm{L} ; \mathrm{t}=\right.$ time; $\mathrm{L}$ = column length, $\mathrm{cm})$. The number of plates for a typical band migrating from the 2D separation (TR, see Figure $4 \mathrm{~A}$ ) was $7.2 \times 10^{5}$, resulting in $\mathrm{H}_{\text {TOT }}=5.6 \times 10^{-6} \mathrm{~cm}$. Therefore, the diffusional component to $\mathrm{H}_{\text {Tот }}$ was calculated to be approximately $46 \%$. For $6110 \mathrm{~s}$ MEKC cycles, which represents the parking time (610 s), and a total separation time of $720 \mathrm{~s}$, the percent contribution of diffusional spreading during the parking phases only to $H_{D}$ is roughly $85 \%$ or $2.2 \times 10^{-6} \mathrm{~cm}$. Clearly, reductions in the development time for the MEKC cycles can reduce $\mathrm{H}_{D}$ resulting from the parking phases of the separation, producing higher peak capacities. Interestingly, the diffusion coefficient for cytochrome $\mathrm{C}$ in free solution has been reported to be $\sim 10^{-6} \mathrm{~cm}^{2} \mathrm{~s}^{-1}, 2$ two-orders of magnitude higher than that in the viscous polyacrylamide sieving matrix. Therefore, using MEKC in the first dimension, where parking is required to allow 
development in the second dimension, would be inadvisable due to significant degradations in peak capacity arising from large values of $\mathrm{H}_{\mathrm{D}}$.

Another process that could add to the total plate height of this coupled 2D separation is protein destacking at the interface of the sieving matrix with the pseudo-stationary phase of the MEKC dimension due to mobility mismatches. Inspection of an injection band produced by a single MEKC cycle (see inset of Figure 4A) resulted in an electrophoretic band width (FWHM) of $0.15 \mathrm{~s}$. The band width obtained solely from the $1 \mathrm{D}$ MEKC run was $0.14 \mathrm{~s}$. Therefore, the coupling process resulted in a $6.7 \%$ increase in the observed $2 \mathrm{D}$ band width. The relatively small increase in the band width resulted from the narrow channel used in the first dimension and the lack of sieving matrix (see Figure 1B) in the path defining the second dimension, which produced acceleration of material entering into the second dimension due to the analytes lower mobility in the first dimension. Both of these observations define the width of the injection plug introduced into the second dimension, which would be $\leq 20 \mu \mathrm{m}$, which is $0.07 \%$ of the total column length for the MEKC dimension.

Orthogonality of SDS $\mu$-CGE and MEKC separation techniques. Since the potential peak capacity of a 2D electropherogram is the arithmetic product of the peak capacities of the constituent dimensions (i.e., SDS $\mu$-CGE and MEKC in this case), extremely large peak capacities can be obtained even if the constituent dimensions produce only modest peak capacities. ${ }^{3} \mathrm{~A} 2 \mathrm{D}$ separation actually generates this theoretically available peak capacity only if the constituent $1 \mathrm{D}$ dimensions are completely orthogonal. A high degree of retention correlation between the dimensions can reduce a $2 \mathrm{D}$ separation to what is, in fact, a 1D separation with peaks distributed along the diagonal of a plot of retention times between the constituent dimensions. ${ }^{4}$ The information content of any multi-dimensional system is the sum of the mean information content of each individual dimension minus the cross-information. ${ }^{5}$ Minimizing crossinformation is therefore important in any multi-dimensional separation. ${ }^{4}$ 
Changes in migration order and migration time can serve as indicators of different separation mechanisms responsible for the migration behavior in each dimension. ${ }^{6}$ In the present case to evaluate the orthogonality of SDS $\mu$-CGE and MEKC, ${ }^{7-9}$ protein migration maps were constructed. To perform this, the migration times $\left(\mathrm{MT}_{\mathrm{i}}\right)$ for all ten proteins were acquired for each separation mode according to the $1 \mathrm{D}$ results. Then, the normalized migration times $\left(\mathrm{MT}_{i, n o r m}\right)$ for both $\mathrm{SDS} \mu$-CGE and MEKC were calculated using the following equation;

$$
M T_{i, \text { norm }}=\left[M T_{i}-M T_{\min }\right] /\left[M T_{\max }-M T_{\min }\right]
$$

where $M T_{\max }$ and $M T_{\min }$ represent the migration times of the most and least retained proteins, respectively, for both the SDS $\mu$-CGE and MEKC electropherograms. The migration times for this investigation were obtained by testing each protein individually, and also confirmed by spiking proteins into both the SDS $\mu$-CGE and MEKC microchip separations. The values of $\mathrm{MT}_{\mathrm{i}, \text { norm }}$ range from 0 to 1 . The normalization serves two purposes: First, it allows for comparison of SDS $\mu$-CGE and MEKC as two different sets of $\mu$-CE data in a uniform $2 \mathrm{D}$ migration space regardless of the absolute migration time values, which can change due to electrophoretic conditions such as electric field and the effective length of the separation channel. Second, it removes the void spaces in the 2D separation plot where no peaks migrated. ${ }^{7}$

Normalized 2D relative migration time plots were constructed and are shown in Figure S1 for the ten investigated proteins. The orthogonality of the two individual dimensions can be qualitatively estimated by looking at the position of each protein on this two-dimensional map with respect to the main diagonal (shown by a dashed line in Figure S1). Figure S1 illustrates that the combination of SDS $\mu$-CGE and MEKC can provide good orthogonality as only two proteins fell along the diagonal ( $\mathrm{AC}$ and $\mathrm{CO})$. 


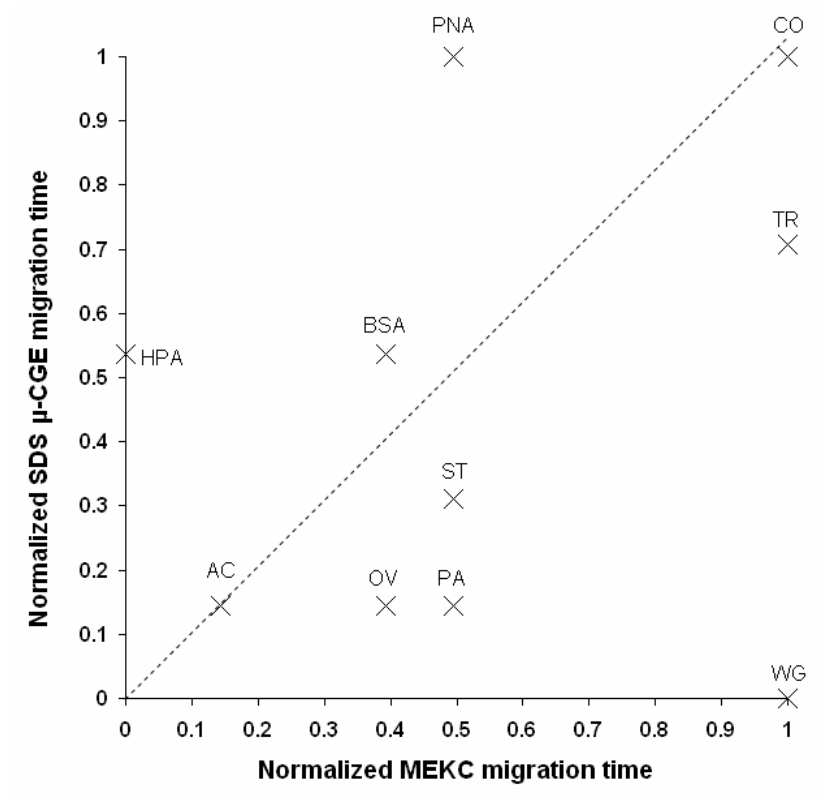

Figure S1. Scatter plot of the normalized migration times for SDS $\mu$-CGE versus MEKC separation dimensions for the investigated proteins. The normalized migration times were calculated using eq. S1 and secured from the data shown in Figures $2 \mathrm{~A}$ and 3.

Geometric Orthogonality of the $2 \mathrm{D}$ separation. Figure S2 represents the 2D separation image (see Figure $4 C)$, which was divided into $10(2 \times 5)$ rectangular bins. See Figure 4 for separation details.

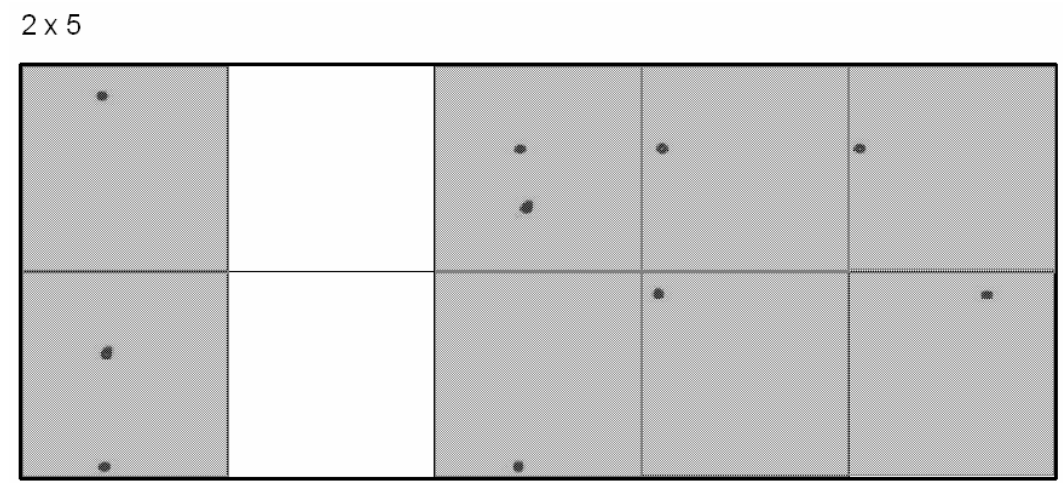

Figure S2. Geometric orthogonality description of the 2D separation of model proteins obtained in a PMMA microchip. 


\section{REFERENCES}

(1) Shadpour, H.; Musyimi, H.; Chen, J.; Soper, S. Journal of Chromatography A 2006, 1111, 238-251.

(2) Lewus, R. K.; Carta, G. Industrial \& Engineering Chemistry Research 2001, 40, 1548-1558.

(3) Cortes, H. J.; Editor Chromatographic Science Series, Vol. 50: Multidimensional Chromatography: Techniques and Applications, 1990.

(4) Venkatramani, C. J.; Xu, J.; Phillips, J. B. Analytical Chemistry 1996, 68, 1486-1492.

(5) Erni, F.; Frei, R. W. Journal of Chromatography 1978, 149, 561-569.

(6) Rocklin, R. D.; Ramsey, R. S.; Ramsey, J. M. Analytical Chemistry 2000, 72, 52445249.

(7) Gilar, M.; Olivova, P.; Daly, A. E.; Gebler, J. C. Analytical Chemistry 2005, 77, 64266434.

(8) Slonecker, P. J.; Li, X. D.; Ridgway, T. H.; Dorsey, J. G. Analytical Chemistry 1996, 68, 682-689.

(9) Gray, M.; Dennis, G. R.; Wormell, P.; Shalliker, R. A.; Slonecker, P. Journal of Chromatography A 2002, 975, 285-297. 